\title{
Changing one cell type into another
}

Two independent groups identify factors that drive direct conversion to oligodendrocyte precursor cells in the rodent.

It has been more than two decades since the first demonstration that one cell type can be converted into another through the ectopic expression of a transcription factor. But what began as an isolated trickle has now grown into a steady stream of reported lineage conversions. Recently, the groups of Paul Tesar at Case Western Reserve University and of Marius Wernig at Stanford University identified factors that can convert fibroblasts into oligodendrocyte precursor cells (OPCs) in the mouse and rat.

As the name suggests, OPCs generate oligodendrocytes, which are the main myelinating cells in the central nervous system. In the context of cell transplantation, OPCs have had ameliorating effects in rodent models of nerve damage and myelin loss and are thus considered a potential therapeutic cell type for human diseases that involve myelin. The present sources of OPCs for cell transplantation, however, are not ideal: fetal OPCs are not available in large numbers and cannot be easily expanded, and although OPCs can be obtained through differentiation of pluripotent stem cells, the methods to do this are still laborious. Direct lineage conversion of other somatic cells to OPCs offers an alternative.

Finding the factors to achieve such lineage conversion is no small matter, however. The experiments in the 1980 s that converted fibroblasts to muscle cells involved overexpressing a single transcription factor, MyoD, a master regulator of skeletal muscle development. But for most cell types, it is not so simple: a combination of transcription factors is likely to be necessary for most direct conversions of cell fate.

Researchers in both the Tesar and Wernig groups, in their work on generating OPCs, combined several types of prior knowledge

to come up with shortlists of factors that are good candidates for promoting OPC fate. Both groups began with genomewide gene expression data, focusing on genes expressed in oligodendrocytes and OPCs but not in cells of other neural lineages. They then whittled down their lists to genes encoding factors known from prior experiments to be functionally involved in oligodendrogenesis, either because expression of the

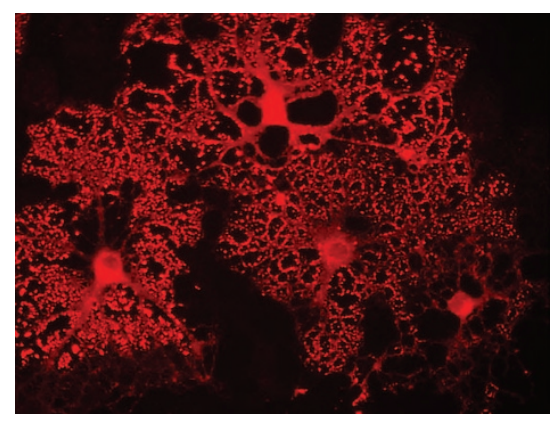

Oligodendrocytes via reprogramming. OPCs obtained by lineage conversion of mouse fibroblasts were differentiated to oligodendrocytes. Reprinted from Nature Biotechnology (Najm et al., 2013). of Nature Methods, Ilya Shmulevich and colleagues at the Institute for Systems Biology, Seattle, make this argument and describe one such approach. In a method based on the logic of developmental binary toggle switches, Shmulevich and colleagues show that, by measuring reversals in gene expression between cell types, they can retrospectively identify transcription factors known to be important for cell-fate decisions as ral progenitors promotes the process or because mutation of the genes results in developmental defects in this lineage.

Tesar and colleagues (Najm et al., 2013) settled on a list of eight factors; Wernig and colleagues (Yang et al., 2013) on an (overlapping) list of ten. What remained then was the systematic and painstaking process of experimental trial and error to identify the simplest combination of factors that can turn fibroblasts into OPCs. For the screening process itself, both groups made use of a green fluorescent reporter for proteolipid protein, which is expressed in both OPCs and oligodendrocytes, infecting embryonic fibroblasts from a transgenic mouse with pools of lentiviruses delivering the candidate reprogramming-factor genes. Tesar and colleagues report that the combination of Sox10, Olig2 and Nkx6.2 does the trick; Wernig and colleagues' preferred combination is Sox10, Olig 2 and Zfp536.

Might informatics approaches to better parse genome-wide, cell type-specific gene expression data ease the laborious process of identifying candidate reprogramming factors? In a paper published in this issue well as for previously reported fate conversion of fibroblasts to neurons.

Of course, simply looking for specifically expressed transcription factors for which there is also functional evidence has served several groups admirably so far. In the work of the Tesar and Wernig groups, the identified factors yielded cells that, as determined by markers and by functional phenotype in vitro and in vivo, behave as bona fide OPCs. But perhaps especially for cell types for which there is little prior functional information available, analysis approaches such as that described by Shmulevich and colleagues-and there will doubtless be others-could prove useful for prioritizing candidate factors for empirical testing.

\section{Natalie de Souza}

\section{RESEARCH PAPERS}

Heinäniemi, M. et al. Gene-pair expression signatures reveal lineage control. Nat. Methods 10, 577-583 (2013).

Najm, F.J. et al. Transcription factor-mediated reprogramming of fibroblasts to expandable, myelinogenic oligodendrocyte progenitor cells. Nat. Biotechnol. 31, 426-433 (2013).

Yang, N. et al. Generation of oligodendroglial cells by direct lineage conversion. Nat. Biotechnol. 31, 434-439 (2013). 